INVARIANT DISTRIBUTIONS FOR MONOTONE

MARKOV PROCESSES

by

Hugo A. Hopenhayn and Edward C. Prescott

Discussion Paper No. 242, September 1987

\author{
Center for Economic Research \\ Department of Economics \\ University of Minnesota \\ Minneapolis, Minn 55455
}




\title{
Invariant Distributions for Monotone Markov Processes*
}

\author{
Hugo A. Hopenhayn and Edward C. Prescott**
}

August, 1986

Revised, September 1987

${ }^{*}$ We acknowledge the helpful discussion and comments of Larry Epstein, Rodolfo Manuelli, Ramon Marimon and Nancy L. Stokey. We thank the Federal Reserve Bank of Minneapolis and the National Science Foundation for financial support.

**Stanford University and University of Minnesota. 


\begin{abstract}
The existence of fixed points for monotone maps on spaces of measures is established. The case of monotone Markov processes is analyzed and a uniqueness and global stability condition is developed. A comparative statics result is presented and the problem of approximation to the invariant distribution is discussed. The conditions of the theorems are verified for the cases of Optimal Stochastic Growth and Industry Equilibrium.
\end{abstract}


Section 1. Introduction.

A problem that is arising with increasing frequency in economic analyses is the study of invariant distributions for time-invariant Markov processes. These arise in at least two classes of problems. The first is when the object of the research is an equilibrium distribution of agents indexed by some economic characteristics such as income, asset holding or employment status. Examples of such applications are the equilibrium search and unemployment studies of Lucas and Prescott(1974) and of Jovanovic(1979), the asset holding problems of Lucas(1978) and Manuelli(1986) and the entry-exit model of Hopenhayn(1986).

The second class are the Markov processes that arise as solutions to dynamic stochastic optimization problems. Examples of these are the optimal stochastic growth model studied by Brock and Mirman(1972) and Donaldson and Mehra(1983) and the multi-capital good generalization of Marimon(1984). There, the question is whether the sequence of predictive probability distributions of future states has a limit and whether this limit is independent of the initial state.

Such analyses have not proven easy. They require considerable investment in specialized mathematics and often considerable ingenuity in verifying conditions of the available theorems. In this paper we develop some easily verifiable conditions for the important class of Markov processes that are monotone in the stochastic sense, that is if the current state is larger, the conditional distribution of tomorrow's state is stochastically larger.

Unlike the Feller-type conditions approach(1), our approach does not require continuity of the process. This is useful when there are nonconvexities that result in a discontinuous optimal policy function. Majumdar, Mitra and Nyarko(1985) prove for an important class of growth models with nonconvexites in the production technology that the optimal policy function is increasing and consequently our theorems can be applied(2) 
Our existence argument applies more generally to the existence of fixed points for monotone mappings of a compact set of measures into itself. The mapping need not be linear and measure preserving as is the case for the Markov process. Besides an existence result a uniqueness condition is developed for monotone Markov processes. It requires only that the process be such that there is some point in the state space with the property that for any initial state the probability of staying strictly above it for ever or strictly below it for ever is less than one.

In section 2 we develop the main fixed point theorem. In section 3 the theorem is applied to Markov processes. Section 4 provides a sufficient condition for the fixed point of the monotone Markov process to be unique and for the sequence obtained by succesive approximations to converge to this fixed point. Section 5 presents two examples of economic applications of the theorems. 
Section 2. A fixed point result

In this section we present our key fixed point result which is of more general interest. Loosely speaking, we will show that monotone maps defined on certain spaces of measures have fixed points. The following definitions will allow us to make the above statement precise.

The underlying structure is an ordered space $(S, \geq)$, i.e. a space with a relation $\geq$, called an order, that satisfies the following properties:
a) reflexivity: $s \in S$ implies $s \geq s$.
b) transitivity: If $s, t$, and $u$ are in $S, s \geq t$ and $t \geq u$ imply $s \geq u$.
c) antisymmetry: If $s$ and $t$ are in $S, s \geq t$ and $t \geq s$ imply $t=s$.

We say that the order $\geq$ is closed in $S$ if $\operatorname{gr}(\geq)=\left\{\left(s, s^{\prime}\right) \in S \times S: s \geq s^{\prime}\right\}$, the graph of $\geq$ on $S$, is a closed subset of $S \times S$. If $S$ is a metric space, as it will be in our case, $\geq$ is closed on $S$ if and only if for every pair of sequences $\left\{s_{n}\right\}$ and $\left\{s_{n}^{\prime}\right\}$ in $S$ with $s_{n} \geq s_{n}^{\prime}, s_{n} \rightarrow s$ and $s_{n}^{\prime} \rightarrow s^{\prime}$ imply $s \geq s^{\prime}$.

An upper(lower) bound for MCS is an element $s \in S$ with $s^{\prime} \leq s\left(s \geq s^{\prime}\right)$ for all $s^{\prime} \in M$. The supremum of $M$, if it exists, is an upper bound for $M$ which is a lower bound for the set of all upper bounds of $\mathrm{M}$. A chain $\mathrm{C}$ on $\mathrm{S}$ is a subset of $\mathrm{S}$ for which all pairs of elements are comparable; i.e. for all $s$ and $s^{\prime}$ in $C, s^{\prime} s^{\prime}$ or $s^{\prime} \geq s$.

Let $A_{\leq}$be the set that includes all elements in $S$ that are smaller than some element in A. i.e. $A_{\leq}=\left\{s^{\prime} \in S: s^{\prime} \leq s\right.$ for some $\left.s \in A\right\}$. Define in the analogous way the set $A_{\geq}$. We will say that $A$ is a decreasing (increasing) set if $A=A_{\leq}\left(A=A_{\geq}\right)$. The set $A$ is said to be monotone if it it is either decreasing or increasing.

A mapping f from an ordered space $(S, \geq)$ to and ordered space $(T, \succeq)$ is said to be a monotone function if for any two elements $s, s^{\prime}$ in $S, s \geq s^{\prime}$ implies $f(s) \succeq f\left(s^{\prime}\right)$. 
Our first basic assumption on the structure of our construction is:

Assumption I. $(\mathrm{S}, \geq)$ is an ordered space were $\mathrm{S}$ is a compact metric space and $\geq \mathrm{a}$ closed order. The measure space considered in this paper will be $(\mathrm{S}, \mathcal{S})$ were $\mathcal{S}$ is the $\sigma$-algebra generated by the open sets on S, i.e. the Borel $\sigma$-algebra (3).

Let $\mathcal{M}(\mathrm{S})$ be the space of finite measures on $(\mathrm{S}, \mathcal{S})$. To define monotonicity we need to provide an order to $\mathcal{M}(\mathrm{S})$. For this purpose we will define a relation $\succeq$ on $\mathcal{M}(\mathrm{S})$ in the following way:

Definition of the stochastic order. For any pair of elements $\mu$ and $\mu^{\prime}$ in $\mathcal{H}(\mathrm{S})$, we will say that $\mu \succeq \mu^{\prime}$ if for every monotone, measurable and bounded function $\mathrm{f}:(\mathrm{S}, \geq) \rightarrow \mathrm{R}_{+}$

$$
\int \mathrm{f} \mu(\mathrm{ds}) \geq \int \mathrm{f} \mu^{\prime}(\mathrm{ds})
$$

Whenever $\mu \succeq \mu^{\prime}$ we will say that $\mu$ stochastically dominates or is stochastically greater than $\mu^{\prime}\left({ }^{4}\right)$. When $\mu$ and $\mu^{\prime}$ are probability measures this coincides with the familiar notion of stochastic dominance used in economics and finance. In particular, when $\mathrm{S}$ is a subset of the real line it is simple to show that $\mu \succeq \mu^{\prime}$ if and only if $\mathrm{F}_{\mu}(\mathrm{s}) \leq \mathrm{F}_{\mu^{\prime}}(\mathrm{s})$ for every $\mathrm{s} \in \mathrm{S}$, where $\mathrm{F}_{\mu}$ and $\mathrm{F}_{\mu^{\prime}}$ are the distribution functions of $\mu$ and $\mu^{\prime}$, respectively. (If $\mathrm{S}$ is a subset of $\mathrm{R}^{\mathrm{n}}$ where $\mathrm{n}>1$, however, $\mathrm{F}_{\mu}(\mathrm{s}) \leq \mathrm{F}_{\mu^{\prime}}(\mathrm{s})$ does not imply $\mu \succeq \mu^{\prime}$. Only the converse is true).

In this section we will show that if $\mathrm{T}$ is a monotone map from $\mathcal{M}(\mathrm{S})$ into itself, $\mathrm{T}$ has a fixed point if and only if there is a measure $\mu_{\mathrm{a}}$ in $\mu(\mathrm{S})$ such that $\mathrm{T}\left(\mu_{\mathrm{a}}\right) \succeq \mu_{\mathrm{a}}$. Since $\succeq$ is reflexive (as will be shown) necessity is immediate.

The proof of sufficiency will be based on the following theorem due to Knaster-Tarski (for a reference see Dugundji and Granas, 1982). 
Theorem (Knaster-Tarski). Let $(\mathrm{P}, \geq)$ be an ordered space and $\mathrm{F}: \mathrm{P} \rightarrow \mathrm{P}$ a monotone function. Assume that there is a $b \in P$ such that both

i. $b \leq F(b)$ and

ii. every chain in $\{x \in P: x \geq b\}$ has a supremum.

Then the set of fixed points of $\mathrm{F}$ is not empty and among them there exists at least one undominated fixed point (i.e.a $\lambda \in P$ such that $F(\lambda)=\lambda$ and no other fixed point $\lambda^{\prime} \neq \lambda$ with $\lambda^{\prime} \geq \lambda$ exists).

In order to use the above theorem it is necessary to show that $\succeq$ is an order and that condition i. above is satisfied (5). For the latter it will be convenient to consider $M(S)$ as a topological space by giving it the relative weak* topology ( which in probability theory is known as the topology of weak convergence of measures). With this topology $M(S)$ is a metric space (6).

With the weak* topology a sequence(or net) of measures $\mu_{\mathrm{n}}$ in $\mathcal{H}(\mathrm{S})$ converges to the measure $\mu$ if and only if $\int \mathrm{f} \mu_{\mathrm{n}}(\mathrm{ds}) \rightarrow \int \mathrm{f} \mu(\mathrm{ds})$ for every continuous and bounded $\mathrm{f}:(\mathrm{S}, \mathcal{S}) \rightarrow \mathrm{R}$. To exploit this fact we would like to be able to restrict the set of functions that determine the stochastic dominance order to the class of nonnegative, continuous and bounded monotone functions. The next two propositions show that this can be done without loss of generality. Before doing so, we will need to introduce some additional notation.

Let $\mathbf{M}=\{\mathrm{f}: \mathrm{S} \rightarrow \mathrm{R}+$ such that $\mathrm{f}$ is monotone, continuous and bounded $\}$. For any $\mu \in \mathcal{M}(\mathrm{S})$ let $L^{1}(\mu)$ be the space of $\mu$-integrable functions on $S$ with norm given by $\|\mathrm{f}\|_{\mu}=\int|\mathrm{f}| \mu(\mathrm{ds})$. We will denote this norm by $\|\mathrm{f}\|$ when the measure $\mu$ is understood. A set $\operatorname{DCL}^{1}(\mu)$ is $\operatorname{L}^{1}(\mu)$ dense in the set $\operatorname{ACL}^{1}(\mu)$ if DCA and if for every function $g$ in $A$ there is a sequence $\left\{f_{n}\right\}$ with $\mathrm{f}_{\mathrm{n}} \in \mathrm{D}$ such that $\left\|\mathrm{f}_{\mathrm{n}}-\mathrm{g}\right\| \rightarrow 0$ or equivalently, for any $\epsilon>0$ there exists an $f \in D$ with $\|\mathrm{g}-\mathrm{f}\|<\epsilon$. 
Proposition 1. For any $\mu \in \mu(\mathrm{S}), M$ is $L^{1}(\mu)$ dense in the class of monotone, nonnegative and bounded measurable functions on $\mathrm{S}$.

Proof. Step 1: Monotone indicator functions are contained in the closure of $\mathbf{M}$. Let $\mathrm{g}$ be a monotone indicator function. Then $g=\chi_{A}$ for some set $A \in \mathcal{S}$. Fix $\epsilon>0$. Since $S$ is a metric space, $\mu$ is a regular measure (see, for example, Parthasarathy, 1967). Thus there exists sets CcS and DCS closed such that CCA ${ }^{\mathrm{C}}$, DCA and $\mu(\mathrm{CUD})>\mu(\mathrm{S})-\epsilon$.

The set $\mathrm{C}_{\leq}$contains $\mathrm{C}$ (by reflexivity) and, as we now show, is closed. Let $\left\{\mathrm{s}_{n}^{\prime}\right\}$ be a sequence in $\mathrm{C}_{\leq}$converging to some element $\mathrm{s}^{\prime} \in \mathrm{S}$. Then there exists a sequence $\left\{\mathrm{s}_{\mathrm{n}}\right\}$ in $\mathrm{C}$ with $s_{n}^{\prime} \leq s_{n}$. Since $C$ is a closed subset of a compact space it is compact and there is a subsequence $\left\{s_{n_{k}}\right\}$ of $\left\{s_{n}\right\}$ that converges to some element $s \in C$. But then $s_{n_{k}}^{\prime} \leq s_{n_{k}}$ and $s_{n_{k}}^{\prime}$ converges to $s^{\prime}$. Consequently $s^{\prime} \leq s$ and thus $s^{\prime} \in C_{\leq}$. This proves that $C_{\leq}$is closed. Similarly one can establish that $D_{\geq}$contains $D$ and is closed. Also $C_{\leq} \cap D_{\geq}=\phi$ since otherwise there would be elements $s \in S$, $a \in C$ and $b \in D$ with $a \geq s \geq b$, so $a \geq b$. But $a \in C \subset A^{c}$ and $b \in D \subset A$ so $g(a)=0<1=g(b)$, $a$ contradiction to the fact that $g$ is monotone.

Since $\mathrm{S}$ is compact and $\geq$ is a closed order, $(\mathrm{S}, \geq)$ is a compact ordered space (Nachbin, 1965). Hence by Theorem 4 of $\S 3$ and Theorem 1 of $\S 2$ in Nachbin (1965) there is a continuous and monotone function $\mathrm{f}$ on $\mathrm{S}$ such that $\mathrm{f}(\mathrm{s})=0$ for $\mathrm{s} \in \mathrm{C}_{\leq}, \mathrm{f}(\mathrm{s})=1$ for $\mathrm{s \in D}$ and $0 \leq \mathrm{f}(\mathrm{s}) \leq 1$ for $\mathrm{s} \in \mathrm{S}(\boldsymbol{\pi})$. In consequence,

$$
\begin{aligned}
\|\mathrm{g}-\mathrm{f}\| & =\int|\mathrm{g}(\mathrm{s})-\mathrm{f}(\mathrm{s})| \mu(\mathrm{ds}) \\
& \leq \int_{\operatorname{CUD}}^{\int}|\mathrm{g}(\mathrm{s})-\mathrm{f}(\mathrm{s})| \mu(\mathrm{ds})+\underset{\operatorname{CUD}^{\mathrm{C}}}{\int \mathrm{g}(\mathrm{s}) \mu(\mathrm{ds})<\epsilon .}
\end{aligned}
$$

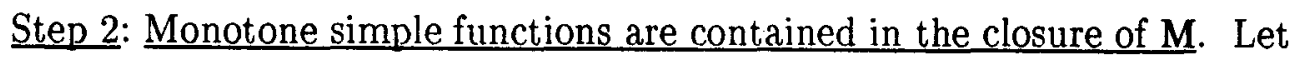

$\mathrm{g}(\mathrm{s})=\sum_{\mathrm{i}=1}^{\mathrm{n}} \mathrm{c}_{\mathrm{i}} x_{\mathrm{A}_{\mathrm{i}}}(\mathrm{s})$ be a monotone simple function. Without loss of generality we can assume $c_{i} \leq c_{j}$ whenever $i \leq j$ and $A_{i} \cap A_{j}=\phi$ for $i \neq j$. Write 


$$
g(s)=\sum_{i=1}^{n}\left(\sum_{j=1}^{i} a_{j}\right) x_{A}(s), \text { where } a_{j}=c_{j}-c_{j-1} \text { and } c_{0}=0 \text {. }
$$

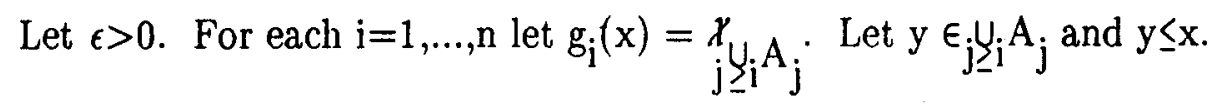

Then $g(x) \geq g(y)$ and thus $x \in \epsilon_{j} \bigcup_{i} A_{j}$. So the functions $g_{j}$ are monotone. Also,

$$
g=\sum_{i=1}^{n}\left(\sum_{j=1}^{i} a_{j}\right) x_{A_{i}}=\sum_{i=1}^{n} a_{j} \underset{j \geq i}{\sum} x_{A_{j}}=\sum_{i=1}^{n} a_{i} g_{i}
$$

By case 1 there exist continuous and monotone functions $f_{i}: S \rightarrow[0,1]$ with $\left\|g_{i}-f_{i}\right\|<\frac{\epsilon}{n c_{n}}$. Let $f=\sum_{i=1}^{n} a_{i} f_{i}$. Since $a_{i} \geq 0, f$ is monotone, continuous and non-negative. Finally

$$
\begin{aligned}
\|g-f\| & =\left\|\Sigma a_{i} g_{i}-\Sigma a_{i} f_{i}\right\|=\left\|\Sigma a_{i}\left(g_{i}-f_{i}\right)\right\| \\
& \leq \Sigma a_{i}\left\|g_{i}-f_{i}\right\|<n c \frac{\epsilon}{n c}=\epsilon .
\end{aligned}
$$

Step 3: $\underline{M}$ is $L^{1}(\mu)$ dense in the class of nonegative monotone, bounded and measurable functions. By the standard proof of denseness of simple functions in $L^{1}(\mu, S)$ (see for theorem 1.5.5 in Ash, 1972), g can be approximated by a monotone simple function. This, together with the results for case 2 , establishes that $\mathrm{g}$ can be approximated by a continuous, monotone and nonnegative function $\square$.

We are now prepared to show that in defining the stochastic order there is no loss in generality by restricting attention to the set of continuous and monotone functions from $S$ to $\mathrm{R}_{+}$.

Proposition 2. For any two measures $\mu$ and $\nu$ in $\mu(\mathrm{S}), \mu \underline{\imath} \nu$ if and only if $\int \mathrm{f} \mu(\mathrm{ds}) \geq \int \mathrm{f} \nu(\mathrm{ds})$ for all $f \in M$, the set of continuous, nonnegative and bounded real valued functions on $S$. 
Proof. Necessity is immediate by definition. Suppose $\mathrm{g}$ is a monotone, bounded and measurable function from $S$ to $R_{+}$. By Proposition 1 there exists a sequence $\left\{f_{n}\right\}, f_{n} \in \mathbf{M}$ with $\lim _{n \rightarrow \infty}$ $\left\|\mathrm{g}-\mathrm{f}_{\mathrm{n}}\right\|_{(\mu+\nu)}=0$. Notice that since $\mathrm{f}_{\mathrm{n}}$ are positive and bounded, $\left\|\mathrm{g}-\mathrm{f}_{\mathrm{n}}\right\|_{\mu}$ and $\left\|\mathrm{g}-\mathrm{f}_{\mathrm{n}}\right\|_{\nu}$ converge to zero as $n \rightarrow \infty$. Suppose $\int \mathrm{g} \mu(\mathrm{ds})<\int \mathrm{g} \nu(\mathrm{ds})$. Then there exists some $\mathrm{N}$ such that for $\mathrm{n} \geq \mathrm{N}$ $\int \mathrm{f}_{\mathrm{n}} \mu(\mathrm{ds})<\int \mathrm{f}_{\mathrm{n}} \nu(\mathrm{ds})$, contradicting the fact that $\int \mathrm{f} \mu(\mathrm{ds}) \geq \int \mathrm{f} \nu(\mathrm{ds})$ for all $\mathrm{f}$ in $\mathbf{M}$.

Having established this result, we next show that $\succeq$ inherits from $\geq$ the properties of a closed order. In proving that $\succeq$ is an order, it is necessary to show that $\succeq$ is antisymmetric; that is, given two measures $\mu$ and $\nu$ in $\mu(\mathrm{S})$ with $\mu \succeq \nu$ and $\nu \succeq \mu$, we need to verify that $\mu=\nu$. (This is equivalent to saying that the class of monotone, nonegative, bounded measurable functions is separating in,$(\mathrm{S}))$. This result, which does not seem obvious to us, is established in Proposition 3. In order to do so, we develop four lemmas. Loosely speaking, these lemmas imply that the monotone sets generate the topology of $\mathrm{S}$ in such a way that if two measures coincide on the monotone sets they do so on all Borel sets.

Lemma 1. If $\mathrm{S}$ is compact and $\geq$ a closed order, the monotone subsets form a subbase for the topology.

Proof. See Theorem $5 . \S 3$ in Nachbin(1965).

Lemma 2. Let $\mathcal{B}$ be a subclass of $\mathcal{S}$ such that (i) $\mathcal{B}$ is closed under the formation of finite intersections and (ii) each open set in $\mathrm{S}$ is a finite or countable union of elements of $\mathcal{B}$. Then $B$ is convergence determining and hence separating.

Proof. See Theorem 2.2 in Billingsley(1968).

Lemma 3. If the measures $\mu$ and $\nu$ in $.4(S)$ agree on monotone sets, then they agree on finite intersections of monotone sets. 
Proof. If $A$ is a decreasing set then $A^{C}$ is increasing, since if $a \in A^{c}$ and $a^{\prime} \geq a$, then $a^{\prime} \in A$ implies $a \in A$, a contradiction to $a \in A^{c}$. Similarly, if $A$ is an increasing set, $A^{c}$ is decreasing.

We will next show that the measures agree on finite intersections of monotone sets. So if $\mathrm{A}$ and $\mathrm{B}$ are two monotone sets, we need to show that $\mu(\mathrm{A} \cap \mathrm{B})=\nu(\mathrm{A} \cap \mathrm{B})$. If $\mathrm{A}$ and $\mathrm{B}$ are both increasing(decreasing), then $\mathrm{A} \cap \mathrm{B}$ will clearly be increasing(decreasing). Hence assume $\mathrm{A}$ is a decreasing set and $B$ an increasing set.

Note that $B \backslash(A \cap B)$ is increasing since if $s \in B \backslash(A \cap B)$ and $s^{\prime} \geq s$, then $s^{\prime} \notin A$; otherwise $s \in(A \cap B)$, so $s^{\prime} \in B \backslash(A \cap B)$. $B^{C}$ was shown to be a decreasing set. $A \cap B=\left[B^{C} \cup B \backslash(A \cap B)\right]^{C}$. Thus $(\mathrm{A} \cap \mathrm{B})=\mu(\mathrm{S})-\left[\mu\left(\mathrm{B}^{\mathrm{C}}\right)+\mu[\mathrm{B} \backslash(\mathrm{A} \cap \mathrm{B})]\right.$.

But $S, B^{C}$ and $B \backslash(A \cap B)$ are monotone sets so

$$
\begin{aligned}
\nu(\mathrm{A} \cap \mathrm{B}) & =\nu(\mathrm{S})-\left[\nu\left(\mathrm{B}^{\mathrm{C}}\right)+\nu[\mathrm{B} \backslash(\mathrm{A} \cap \mathrm{B})]\right. \\
& =\mu(\mathrm{S})-\left[\mu\left(\mathrm{B}^{\mathrm{C}}\right)+\mu[\mathrm{B} \backslash(\mathrm{A} \cap \mathrm{B})]=\mu(\mathrm{A} \cap \mathrm{B}) .\right.
\end{aligned}
$$

Any finite intersection of monotone sets can be easily be checked to be an intersection of two monotone sets $\square$.

Lemma 4. The stochastic dominance relation $\succeq$ is antisymmetric,i.e. if $\mu$ and $\nu$ are two measures in $\varkappa(\mathrm{S})$, then $\mu \succeq \nu$ and $\nu \succeq \mu$ imply $\mu=\nu$.

Proof. If $\mathrm{A}$ is a monotone set, then $x_{\mathrm{A}}$, the indicator function of the set $\mathrm{A}$, is monotone and thus $\mu(\mathrm{A})=\int x_{\mathrm{A}} \mu(\mathrm{ds})=\int x_{\mathrm{A}} \nu(\mathrm{ds})=\nu(\mathrm{A})$. Consequently by Lemma $3, \mu$ and $\nu$ coincide on finite intersections of monotone sets. Since by Lemma 1 monotone sets form a subbase for the topology, $\mu$ and $\nu$ coincide on a base for the topology, which we will denote by $\mathcal{B}$. Since $\mathrm{S}$ is a compact metric space it has a countable base (sce Theorem I.6.19 in Dunford and Schwartz, 1958). Hence, by the Lindelöf covering theorem (see theorem 15 of chapter I in Kelley, 1955), any open set, in $S$ can be expressed as a countable union of members of $\mathcal{B}$. 
But then $\mathcal{B}$ satisfies the assumptions of Lemma 2 and since $\mu$ and $\nu$ agree on $\mathcal{B}$ we may conclude that $\mu=\nu$ a.

Proposition 3 . The relation $\succeq$ defined on $\mathcal{H}(\mathrm{S})$ is a closed order.

Proof. Reflexivity and transitivity of $\succeq$ are immediate. By Lemma 4 , $\succeq$ is antisymmetric and hence an order. To show that $\succeq$ is closed, let $\left\{\mu_{n}\right\}$ and $\left\{\nu_{n}\right\}$ be two sequences in $\nVdash(S)$ such that $\mu_{\mathrm{n}} \succeq \nu_{\mathrm{n}}$ for all $\mathrm{n}, \mu_{\mathrm{n}} \rightarrow \mu$ and $\nu_{\mathrm{n}} \rightarrow \nu$, where $\mu$ and $\nu$ are in $\mathcal{M}(\mathrm{S})$. For $\mathrm{f} \in \mathbf{M}, \int \mathrm{f} \mu_{\mathrm{n}}(\mathrm{ds}) \geq \int \mathrm{f} \nu_{\mathrm{n}}(\mathrm{ds})$ for all $\mathrm{n}$, $\int f \mu_{n}(d s) \rightarrow \int f \mu(d s)$ and $\int f \nu_{n}(d s) \rightarrow \int f \nu(d s)$ since $f$ is continuous and bounded. But then $\int f \mu(d s) \geq-$ $\int \mathrm{f} \nu(\mathrm{ds})$. Since $\mathrm{f}$ was chosen arbitrarily, we can conclude that $\mu \succeq \nu$ and hence $\succeq$ is a closed order ㅁ.

We have established that $\succeq$ is an order. To apply the Knaster-Tarski Theorem we also need to establish that every chain in $\mathcal{H}(\mathrm{S})$ has a supremum. This is not true unless we further restrict the space of measures considered. For this purpose we introduce the following assumption.

Assumption II. For the remainder of this section we will restrict the space of measures to a compact subset of $\mathcal{H}(\mathrm{S})$ which we will denote by $\Lambda(S)$.

Some examples of compact subsets of $\mu(\mathrm{S})$ encountered in economic problems are the following:

a) The space of uniformly bounded measures, i.e. $\{\mu \in \mathcal{M}(\mathrm{S})$ such that $\mu(\mathrm{S}) \leq \mathrm{m}\}$ where $\mathrm{m}$ is an upper bound fixed for all $\mu(8)$.

b) Any closed subset of the above, e.g. the space of probability measures.

c) Intervals of measures in the stochastic order, i.e. given to measures $\mu_{\mathrm{a}} \underline{\underline{\mathrm{b}}}$, the set $\left[\mu_{\mathrm{a}}, \mu_{\mathrm{b}}\right]=\left\{\mu \in \mathcal{M}(\mathrm{S}): \mu_{\mathrm{a}} \preceq \mu \preceq \mu_{\mathrm{b}}\right\}$. This is clearly a closed set by Proposition 3 . Since the constant function $\mathrm{f}=1$ is monotone, it is easy to see that $\mu(\mathrm{S}) \leq \mu_{\mathrm{b}}(\mathrm{S})$. Hence $\left[\mu_{\mathrm{a}}, \mu_{\mathrm{b}}\right]$ is a closed subset of 
a uniformly bounded set of measures.

d) Given a continuous function $p: S \rightarrow R$ and two real numbers $a$ and $b$, the set of measures that satisfy $\mathrm{a} \leq \int \mathrm{p}(\mathrm{s}) \mu(\mathrm{ds}) \leq \mathrm{b}$ is closed. If, further, $\mathrm{p}(\mathrm{s}) \geq \epsilon>0$ then $\mu(\mathrm{S}) \leq \mathrm{m}=\frac{\mathrm{b}}{\epsilon}$, and the above set is compact.

In the next Proposition we will use the notion of a generalized sequence or net. This is a generalization of the notion of sequences by allowing the index set, called a directed set, to be of more general nature than the integers. In particular, a chain is a net directed (indexed) by itself and all the notions that will be used below are the familiar notions corresponding to sequences where instead of saying 'there exists an $N$ such that for all $n \geq N$....' we say 'there exists some $\mu^{\prime}$ ' in the chain such that for all $\mu$ in the chain 'after' $\mu^{\prime}$, i.e. $\mu \succeq \mu^{\prime}, \ldots$ '.. (For a good reference see Kelley, 1955).

Proposition 4. Every chain $\mathrm{C}$ in the compact set of measures $\Lambda(\mathrm{S})$ has a supremum. Furthermore, the chain converges to the supremum.

Proof. Since $\Lambda(S)$ is compact, there exists a subnet $C^{\prime}$ that converges to some element $\mu^{*}$ in $\Lambda(\mathrm{S})$. We will now show that $\mu^{*}$ is the supremum of $\mathrm{C}$.

We show first that $\mu^{*}$ is an upper bound for $\mathrm{C}$. For this purpose let $\nu \in \mathrm{C}$. Let $\mathrm{C}^{\prime \prime}=$ $\left\{\mu \in C^{\prime}: \mu \succeq \nu\right\}$. $C^{\prime \prime}$ is a subnet of $C^{\prime}$ so it also converges to $\mu^{*}$. Since $\succeq$ is a closed order, as establișhed in Proposition 3, we can conclude that $\mu^{*} \succeq \nu$.

We will now show that $\mu^{*}$ is the least upper bound. Suppose to the contrary that $\nu^{*}$ is another upper bound and that there exists some $\mathrm{f} \in \mathbf{M}$ with $\int \mathrm{f} \nu^{*}(\mathrm{ds})<\int \mathrm{f} \mu \mu^{*}(\mathrm{ds})$. Since $\mathrm{C}^{\prime}$ converges to $\mu^{*}$, there exists some measure $\mu \in \mathrm{C}^{\prime}$ with $\int \mathrm{f} \nu^{*}(\mathrm{ds})<\int \mathrm{f} \mu(\mathrm{ds})$. This contradicts $\nu^{*}$ being an upper bound for $\mathrm{C}$.

Finally, we show that $\mathrm{C}$ converges to $\mu^{*}$. Suppose, to the contrary, that it did not converge. Then there exists an open set $U$, such that $\mu^{*} \in U$ and for every $\mu$ in $C$ there exists some 
$\mu^{\prime}$ in $\mathrm{C}$ with $\mu^{\prime} \succeq \mu$ and $\mu^{\prime} \in \mathrm{U}^{\mathrm{C}}$. Let $\mathrm{C}^{\prime}=\mathrm{C} \cap \mathrm{U}^{\mathrm{C}}$. Set $\mathrm{C}^{\prime}$ is a subnet of $\mathrm{C}$. Again using compactness, $\mathrm{C}^{\prime}$ has a further subnet that converges to some element $\mu^{\prime} \in \Lambda(\mathrm{S})$. As seen previously $\mu^{\prime}$ is a sup for $\mathrm{C}$, and thus $\mu^{\prime} \succeq \mu^{*}$ and $\mu^{*} \succeq \mu^{\prime}$. Thus, by antisymmetry of $\succeq$ (Lemma 4) $\mu^{\prime}=\mu^{*}$, contradicting the definition of $C^{\prime} \square$.

We now present the main result of this section.

Theorem 1. If $\mathrm{T}: \Lambda(\mathrm{S}) \rightarrow \Lambda(\mathrm{S})$ is monotone relative to $\succeq$, where $\mathrm{S}$ and $\Lambda(\mathrm{S})$ satisfy Assumption I and Assumption II, then $\mathrm{T}$ has a fixed point if and only if there exists a measure $\mu_{\mathrm{a}}$ in $\Lambda(\mathrm{S})$ such that $\mathrm{T} \mu_{\mathrm{a}} \succeq \mu_{\mathrm{a}}$

Proof. Since $\succeq$ is reflexive, necessity is immediate. To prove sufficiency we apply the KnasterTarski theorem. We have proved that $\succeq$ is an order (Proposition 3) and that any chain in $\Lambda(S)$ has a supremum (Proposition 4). Hence $\mathrm{T} \mu_{\mathrm{a}} \succeq \mu_{\mathrm{a}}$ implies that there exists a fixed point for $\mathrm{T} \square$.

A natural question that arises is what happens if $\mathrm{T}^{\mathbf{n}}$ (the composition of $\mathrm{T} \mathrm{n}$ times with itself) rather than $T$, satisfies the hypothesis of Theorem 1. In that case there is a fixed point for $\mathrm{T}^{\mathrm{n}}$. If, additionally, $\mathrm{T}$ is lineär, then it has a fixed point:

Corollary 1. If $\mathrm{T}: \Lambda(\mathrm{S}) \rightarrow \Lambda(\mathrm{S})$ is a linear mapping, if $\mathrm{T}^{\mathrm{n}}$ is monotone and $\mathrm{T}^{\mathrm{n}} \mu_{\mathrm{a}} \succeq \mu_{\mathrm{a}}$ for some measure $\mu_{\mathrm{a}}$ in $\Lambda(\mathrm{S})$, and if Assumptions I and II are satisfied, then $\mathrm{T}$ has a fixed point.

Proof. Since $\mathrm{T}^{\mathrm{n}}$ satisfies the assumptions of Theorem 1 , it has a fixed point, say $\mu_{\mathrm{o}}$. Let $\mu_{\mathrm{k}}=$ $\mathrm{T}^{\mathrm{k}} \mu_{\mathrm{o}}$; then $\mathrm{T} \mu_{\mathrm{k}}=\mu_{\mathrm{k}+1}$ for $\mathrm{k}=0,1, . . \mathrm{n}-1$.

Let $\mu=\frac{1}{\mathrm{n}} \sum_{\mathrm{k}=0}^{\mathrm{n}-1} \mu_{\mathrm{k}}$. Then $\mathrm{T} \mu=\frac{1}{\mathrm{n}} \sum_{\mathrm{k}=0}^{\mathrm{n}-1} \mathrm{~T} \mu_{\mathrm{k}}=\frac{1}{\mathrm{n}} \sum_{\mathrm{k}=1}^{\mathrm{n}} \mu_{\mathrm{k}}=\frac{1}{\mathrm{n}} \sum_{\mathrm{k}=0}^{\mathrm{n}-1} \mu_{\mathrm{k}}$ since $\mu_{\mathrm{n}}=\mu_{\mathrm{o}}$.

Thus $\mathrm{T} \mu=\mu$ and the proof is complete $\square$. 
Let $\mathcal{P}(\mathrm{S})$ be the set of probability measures on $\mathrm{S}$, i.e. $\mathcal{P}(\mathrm{S})=\{\mu \in \mathcal{M}(\mathrm{S}): \mu(\mathrm{S})=1\}$. This will be the space of measures considered in the applications to Markov Processes of the next section. The following corollary will prove very useful.

Corollarv 2. If $\mathrm{T}: \mathcal{P}(\mathrm{S}) \rightarrow \mathcal{P}(\mathrm{S})$ is monotone and $\mathrm{S}$ has a minimum element, (i.e. there exits a $\in \mathrm{S}$ such that $s \geq a$ for all $s \in S$ ) and Assumptions I and II are satisfied, then $T$ has a fixed point. Proof. Let $\delta_{\mathrm{a}}$ be the measure that assigns probability one to the point set $\{a\}$. Then for all $\mu \in \mathcal{P}(\mathrm{S}), \mu \succeq \delta_{\mathrm{a}}$. Hence for any monotone mapping $\mathrm{T}: \mathcal{P}(\mathrm{S}) \rightarrow \mathcal{P}(\mathrm{S})$ it is the case that $\mathrm{T} \delta_{\mathrm{a}} \succeq \delta_{\mathrm{a}}$. Thus in this case any monotone map has a fixed point $\square$.

It is of interest in many economic applications to analyze how changes in some underlying parameters of the economy result in changes in the set of invariant distributions corresponding to these economies. For this purpose, Corollary 3 provides a useful result which, loosely speaking, establishes that if the mappings $\mathrm{T}$ and $\mathrm{T}^{\prime}$ on $\Lambda(\mathrm{S})$ are 'ordered', the set of invariant measures corresponding to them will also be 'ordered'. To make this statement precise, we will say that $T^{\prime}$ dominates $T$ if for all $\mu \in \Lambda(S), T^{\prime} \mu \succeq T \mu$.

Corollary 3 . If $\mathrm{T}^{\prime}$ and $\mathrm{T}$ are two mappings on $\Lambda(\mathrm{S})$ that satisfy all the assumptions of Theorem 1 and $\mathrm{T}^{\prime}$ dominates $\mathrm{T}$, then for every fixed point $\mu$ of $T\left(\mu^{\prime}\right.$ of $\left.\mathrm{T}^{\prime}\right)$ there exists a fixed point $\mu^{\prime}$ of $T^{\prime}(\mu$ of $\mathrm{T})$ such that $\mu^{\prime} \succeq \mu$.

Proof: Let $\mu$ be a fixed point for $\mathrm{T}$. Let $\Lambda^{\prime}(\mathrm{S})=\left\{\mu^{\prime} \in \Lambda(\mathrm{S}): \mu^{\prime} \succeq \mu\right\}$. This is a closed subset of $\Lambda(\mathrm{S})$ and hence it is compact. For any $\mu^{\prime} \in \Lambda^{\prime}(S), T^{\prime} \mu^{\prime} \succeq T^{\prime} \mu \succeq \mu$ and hence $T^{\prime}: \Lambda^{\prime}(S) \rightarrow \Lambda^{\prime}(S)$. By Theorem $1, T^{\prime}$ has a fixed point in $\Lambda^{\prime}(S)$. For any fixed point $\mu^{\prime}$ of $T^{\prime}$ the existence of a fixed point $\mu$ of $\mathrm{T}$ with $\mu \preceq \mu^{\prime}$ can be established in the same way $\square$.

This result is particularly useful when the fixed points of $T$ and $T^{\prime}$ are unique. 
Section 3. Applications to Markov Processes.

In many economic applications, it is of interest to know if the variables that describe the state of the economy at each point in time (state vector) have an invariant distribution, when the state vector follows a stationary Markov process.

In the next corollary we present conditions on the transition function for the Markov Process that guarantee the existence of a stationary distribution. Before that we need to define the mapping $\mathrm{T}: \mathcal{P}(\mathrm{S}) \rightarrow \mathcal{P}(\mathrm{S})$ induced by the Markov process.

Let $\mathrm{P}: \mathrm{S} \times \mathcal{S} \rightarrow[0,1]$ be a transition function describing the Markov Process. We will say that $P$ is increasing if $P$ is increasing in its first argument in the stochastic order sense, i.e. $s$ and $s^{\prime}$ in $S$ and $s \geq s^{\prime}$ imply $P(s,.) \succeq P\left(s^{\prime},.\right)$.

The transition function $P$ induces a mapping $T: \mathcal{P}(S) \rightarrow \mathcal{P}(S)$ defined by

$$
\mathrm{T} \mu(\mathrm{A})=\int \mathrm{P}(\mathrm{s}, \mathrm{A}) \mu(\mathrm{ds})
$$

Corollary 4. If $\mathrm{S}$ is a compact metric space with a minimum element and $\mathrm{P}: \mathrm{S} \times \mathcal{S} \rightarrow[0,1]$ is an increasing transition function, then the Markov Process corresponding to $\mathrm{P}$ has a stationary distribution, i.e. there exists a fixed point for the mapping $\mathrm{T}$ induced by the process.

Proof. By Corollary 2, it suffices to show that $\mathrm{T}$ is monotone. For this purpose, let $\mu$ and $\mu^{\prime}$ be in $\mathcal{P}(\mathrm{S})$ and suppose $\mu^{\prime} \succeq \mu$. Let $\mathrm{f}$ be any monotone, nonnegative, bounded and measurable function. Since monotone indicator functions are dense in the monotone functions of $\mathrm{L}^{1}(\mathrm{~T} \mu)$ and $\mathrm{L}^{1}\left(\mathrm{~T} \mu^{\prime}\right)$ we may assume without loss of generality that $\mathrm{f}=\mathcal{X}_{\mathrm{A}}$, a monotone indicator function. For $s^{\prime} \geq s$, and since $A$ is an increasing set and $P$ is increasing, it is immediate that $\mathrm{P}\left(\mathrm{s}^{\prime}, \mathrm{A}\right) \geq \mathrm{P}(\mathrm{s}, \mathrm{A})$. Hence $\mathrm{P}(., \mathrm{A})$ is a monotone, nonnegative, bounded and measurable function 
too. Then if $\mu^{\prime} \succeq \mu$,

$$
\begin{aligned}
\int \mathrm{f} \mathrm{T} \mu^{\prime}(\mathrm{ds}) & =\mathrm{T} \mu^{\prime}(\mathrm{A})=\int \mathrm{P}(\mathrm{s}, \mathrm{A}) \mu^{\prime}(\mathrm{ds}) \\
& \geq \int \mathrm{P}(\mathrm{s}, \mathrm{A}) \mu(\mathrm{ds})=\mathrm{T} \mu(\mathrm{A})=\int \mathrm{f} \mathrm{T} \mu(\mathrm{ds}) .
\end{aligned}
$$

Hence $\mathrm{T} \mu^{\prime} \succeq \mathrm{T} \mu$ so $\mathrm{T}$ is monotone and the proof is complete $\square$.

In many economic applications the Markov process that the state vector follows is generated in the following manner:

Given a state space $\mathrm{S}$ and a random variable $\epsilon$ defined on a measure space $(\mathrm{E}, \mathcal{E})$ with distribution $\psi$, the evolution of the state is described by a mapping $\mathrm{g}: \mathrm{S} \times \mathrm{E} \rightarrow \mathrm{S}$ with the following interpretation: If at time $\mathrm{t}$ the state vector is $\mathrm{s}_{\mathrm{t}}$ and if the realization of $\epsilon$ is $\epsilon_{\mathrm{t}}$, then $s_{t+1}=g\left(s_{t}, \epsilon_{t}\right)$. This structure induces a mapping $P: S \times \mathcal{S} \rightarrow[0,1]$ defined by

$$
\mathrm{P}(\mathrm{s}, \mathrm{A})=\psi\{\epsilon: \mathrm{g}(\mathrm{s}, \epsilon) \in \mathrm{A}\},
$$

which under appropriate conditions is a transition function for a Markov process. The following lemma provides such conditions.

Lemma 5. If $\mathrm{f}$ is measurable in $\mathrm{S} \times \mathrm{E}$ (with the product $\sigma$-algebra), then the function $\mathrm{P}$ described in (1) is a transition function for a Markov process.

Proof. Measurability of $\mathrm{P}(., \mathrm{A})$ for each $\mathrm{A} \in \mathrm{S}$ follows directly from a special case of Lemma 5.5 in Futia[1982]. For $A \in S$, recall that $P(s, A)=\psi\{\epsilon: g(s, \epsilon) \in A\}$. Since $\psi$ is a probability function and $\mathrm{g}(\mathrm{s},$.$) is measurable, and given that taking inverses under \mathrm{g}(\mathrm{s},$.$) preserves the set the-$ oretic operations, $\mathrm{P}(\mathrm{s},$.$) is a probability function \mathrm{\square}$. 
We can now state and prove our last result in this section.

Corollarv 5. Suppose the function $\mathrm{g}$ in (1) is jointly measurable and monotone in its first argument, and $\mathrm{S}$ is a compact metric space with a minimum element. Then the induced Markov process has a stationary distribution.

Proof. By Corollary 4 it suffices to show that the induced transition function $\mathrm{P}$ is increasing. For this purpose let $s^{\prime} \geq s$ and suppose $A$ is an increasing subset of $S$. Then it suffices to show that $\mathrm{P}\left(\mathrm{s}^{\prime}, \mathrm{A}\right) \geq \mathrm{P}(\mathrm{s}, \mathrm{A})$. Let $\mathrm{A}_{\mathrm{s}}=\{\epsilon \in \mathrm{E}: \mathrm{g}(\mathrm{s}, \epsilon) \in \mathrm{A}\}$ and define $\mathrm{A}_{\mathrm{s}^{\prime}}$, similarly, so $\mathrm{A}_{\mathrm{s}}$ is the section of $\mathrm{g}^{-1}(\mathrm{~A})$ at $s$. It suffices to show that $\mathrm{A}_{\mathrm{S}} \subset \mathrm{A}_{\mathrm{S}^{\prime}}$. Pick $\epsilon \in \mathrm{A}_{\mathrm{S}}$; then $\mathrm{g}(\mathrm{s}, \epsilon) \in \mathrm{A}$. But then $\mathrm{g}(\mathrm{s}, \epsilon) \leq \mathrm{g}\left(\mathrm{s}^{\prime}, \epsilon\right) \in \mathrm{A}$ since $\mathrm{A}$ is an increasing set $\square$. 
Section 4. Convergence to the Unique Invariant Distribution

In this section we provide a simple easily verified condition under which the invariant distribution for the process is unique and globally stable. An algorithm for successively approximating the invariant distribution is also provided (9).

Theorem 2 Suppose $\mathrm{P}$ is increasing, there is a greatest element $\mathrm{b}$ in $\mathrm{S}$, i.e. $\quad \mathrm{b} \geq \mathrm{s}$ for all $s \in S$, and the following condition is satisfied:

Monotone Mixing Condition: There exists a point $\mathrm{s}^{*} \in \mathrm{S}$, an $\epsilon>0$ and an $\mathrm{N}$ such that $\mathrm{P}^{\mathrm{N}}\left(\mathrm{b},\left[\mathrm{a}, \mathrm{s}^{*}\right]\right)>\epsilon$ and $\mathrm{P}^{\mathrm{N}}\left(\mathrm{a},\left[\mathrm{s}^{*}, \mathrm{~b}\right]\right)>\epsilon$.

Then there is a unique stationary distribution $\lambda^{*}$ for process $\mathrm{P}$ and for any initial measure $\mu, \mathrm{T}^{\mathrm{n}} \mu=\int \mathrm{P}^{\mathrm{n}}(\mathrm{s},.) \mu(\mathrm{ds})$ converges to $\lambda^{*}$.

Proof. Choose $\mathrm{N}$ and $\epsilon>0$ satisfying the above condition. Let $\delta_{\mathrm{S}}$ indicate the probability measure that concentrates all the mass on the point set $\{s\}$. We will prove that the following inequality holds:

$$
(1-\epsilon) \delta_{\mathrm{a}}+\epsilon \delta_{\mathrm{s}^{*}} \preceq \mathrm{T}^{\mathrm{N}} \delta_{\mathrm{a}} \preceq \mathrm{T}^{\mathrm{N}} \delta_{\mathrm{b}} \preceq(1-\epsilon) \delta_{\mathrm{b}}+\epsilon \delta_{\mathrm{s}^{*}}
$$

For this purpose, let $f$ denote an arbitrary element of $M$. Then

$$
\int \mathrm{f}(\mathrm{s}) \mathrm{T}^{\mathrm{N}} \delta_{\mathrm{a}}(\mathrm{ds}) \geq \mathrm{f}(\mathrm{a}) \underset{s<\mathrm{s}^{*}}{\int} \mathrm{T}^{\mathrm{N}} \delta_{\mathrm{a}}(\mathrm{ds})+\mathrm{f}\left(\mathrm{s}^{*}\right) \int_{\mathrm{s} \geq \mathrm{s}^{*}} \mathrm{~T}^{\mathrm{N}} \delta_{\mathrm{a}}(\mathrm{ds}) .
$$

Given that $\mathrm{f}(\mathrm{a}) \leq \mathrm{f}\left(\mathrm{s}^{*}\right)$ and $\int \mathrm{T}^{\mathrm{N}} \delta_{\mathrm{a}}(\mathrm{ds})>\epsilon$, this in turn implies 


$$
\begin{aligned}
\int f(s) T^{N} \delta_{a}(d s) & \geq f(a)(1-\epsilon)+f\left(s^{*}\right) \epsilon \\
& =\int f(s)\left\{(1-\epsilon) \delta_{a}+\epsilon \delta_{s^{*}}\right\}(d s) .
\end{aligned}
$$

This establishes the left hand side inequality; the right hand side can be proved in the same way. Since $\mathrm{T}$ is increasing, by induction $\mathrm{T}^{\mathrm{k}}$, the composition of $\mathrm{T}$ with itself $\mathrm{k}$ times, is increasing for $\mathrm{k}=1,2, \ldots$ In particular, $\mathrm{T}^{\mathrm{N}} \delta_{\mathrm{a}} \preceq \mathrm{T}^{\mathrm{N}} \delta_{\mathrm{b}}$, which establishes (1). Using the monotonicity and linearity of $\mathrm{T}^{\mathrm{k}}$ we obtain:

(2) $(1-\epsilon) \mathrm{T}^{\mathrm{k}} \delta_{\mathrm{a}}+\epsilon \mathrm{T}^{\mathrm{k}} \delta_{\mathrm{s}^{*}} \preceq \mathrm{T}^{\mathrm{k}+\mathrm{N}} \delta_{\mathrm{a}} \preceq \mathrm{T}^{\mathrm{k}+\mathrm{N}} \delta_{\mathrm{b}} \preceq(1-\epsilon) \mathrm{T}^{\mathrm{k}} \delta_{\mathrm{b}}+\epsilon \mathrm{T}^{\mathrm{k}} \delta_{\mathrm{S}^{*}}$

for any positive integer $\mathrm{k}$.

By Proposition 4, the monotone sequences $\left\{\mathrm{T}^{\mathrm{k}} \delta_{\mathrm{a}}\right\}$ and $\left\{\mathrm{T}^{\mathrm{k}} \delta_{\mathrm{b}}\right\}$ converge. Let these limits be $\lambda_{a}$ and $\lambda_{b}$ respectively. Since the stochastic order has closed graph (as established in Proposition 3), we obtain:

$$
\lambda_{a} \preceq \lambda_{b}
$$

Since $\mathrm{S}$ is compact, the space of probability measures on $(\mathrm{S}, \mathcal{S})$ is compact so there exists a converging subsequence of $\left\{\mathrm{T}^{\mathrm{k}+\mathrm{N}} \delta_{\mathrm{S}}\right\}$, whose limit will be denoted by $\lambda^{*}$. Hence

$$
(1-\epsilon) \lambda_{\mathrm{a}}+\epsilon \lambda^{*} \preceq \lambda_{\mathrm{a}} \preceq \lambda_{\mathrm{b}} \preceq(1-\epsilon) \lambda_{\mathrm{b}}+\epsilon \lambda^{*} .
$$

For $f \in M$, the above inequality implies

$$
\int \mathrm{f} \lambda_{\mathrm{a}}(\mathrm{ds}) \geq(1-\epsilon) \int \mathrm{f} \lambda_{\mathrm{a}}(\mathrm{ds})+\epsilon \int \mathrm{f} \lambda^{*}(\mathrm{ds})
$$

or that

$$
\int \mathrm{f} \lambda_{\mathrm{a}}(\mathrm{ds}) \geq \int \mathrm{f} \lambda^{*}(\mathrm{ds}) .
$$


This in turn implies $\lambda_{a} \geq \lambda^{*}$. Similarly $\lambda_{b} \leq \lambda^{*}$ so that

$$
\lambda_{a} \geq \lambda^{*} \geq \lambda_{b}
$$

Using the transitivity and antisymmetry of this order (as established in Proposition 3), (3) and (4) imply

$$
\lambda_{a}=\lambda^{*}=\lambda_{b}
$$

We will now show that $\lambda^{*}$ is the unique invariant measure and that from any initial measure the process converges to $\lambda^{*}$. By Theorem 1 there exists a probability measure $\lambda$ such that $\mathrm{T} \lambda=\lambda$. Since $\delta_{\mathrm{a}} \leq \lambda \leq \delta_{\mathrm{b}}$ and $\mathrm{T}^{\mathrm{k}}$ is monotone, $\mathrm{T}^{\mathrm{k}} \delta_{\mathrm{a}} \leq \mathrm{T}^{\mathrm{k}} \lambda=\lambda \leq \mathrm{T}^{\mathrm{k}} \delta_{\mathrm{b}}$. Taking limits this yields $\lambda^{*} \leq \lambda \leq \lambda^{*}$, or $\lambda=\lambda^{*}$. Consequently the fixed point for $\mathrm{T}$ is unique.

This same argument can be applied to any initial probability measure $\mu$, obtaining $\mathrm{T}^{\mathrm{k}} \delta_{\mathrm{a}}$ $\leq \mathrm{T}^{\mathrm{k}} \mu \preceq \mathrm{T}^{\mathrm{k}} \delta_{\mathrm{b}}$. Suppose $\mathrm{T}^{\mathrm{k}} \mu$ did not converge to $\lambda^{*}$. Then there would be a subsequence converging to some probability measure $\lambda \neq \lambda^{*}$. But given that $\succeq$ is a closed order, and that $\mathrm{T}^{\mathrm{k}} \delta_{\mathrm{a}}$ and $\mathrm{T}^{\mathrm{k}} \delta_{\mathrm{b}}$ converge to $\lambda^{*}$, it must be the case that $\lambda^{*} \leq \lambda \leq \lambda^{*}$. But since the stochastic order is antisymmetric, $\lambda=\lambda^{*} \square$.

We have established that a monotone Markov process that satisfies the Monotone Mixing Condition will converge, from any initial distribution, to its unique invariant measure. Furthermore, there is a sense in which the invariant distribution can be successively approximated. From equation (2) and given that $\delta_{\mathrm{b}} \succeq \delta_{\mathrm{a}}$ the following can be deduced:

$$
\mathrm{T}^{\mathrm{kN}} \delta_{\mathrm{b}}-\mathrm{T}^{\mathrm{kN}} \delta_{\mathrm{a}} \preceq(1-\epsilon)^{\mathrm{k}}\left(\delta_{\mathrm{b}}-\delta_{\mathrm{a}}\right)
$$

For any nondecreasing, nonnegative, bounded and measurable function $\mathbf{g}$, 
(6) $\quad 0 \leq \int \mathrm{g} \lambda^{*}(\mathrm{ds})-\int \mathrm{g} \mathrm{T}^{\mathrm{kN}} \delta_{\mathrm{a}}(\mathrm{ds}) \leq \int \mathrm{g} \mathrm{T} \mathrm{T}^{\mathrm{kN}} \delta_{\mathrm{b}}(\mathrm{ds})-\int \mathrm{g} \mathrm{T}^{\mathrm{kN}} \delta_{\mathrm{a}}(\mathrm{ds})$

Combining (5) and (6) we obtain
$0 \leq \int \mathrm{g} \lambda^{*}(\mathrm{ds})-\int \mathrm{g} \mathrm{T}^{\mathrm{kN}} \delta_{\mathrm{a}}(\mathrm{ds}) \leq(1-\epsilon)^{\mathrm{k}}[\mathrm{g}(\mathrm{b})-\mathrm{g}(\mathrm{a})]$

Thus if $\epsilon$ and $\mathrm{N}$ were known, for any given monotone function we could obtain an approximation as close as desired to the expectation of the function with respect to the invariant distribution. 
Section 5. Applications.

Lucas and Prescott(1971) analyze the dynamic stochastic competitive equilibrium of an industry. The inverse demand function $D\left(q_{t}, z_{t}\right)$ is assumed to be continuous, decreasing in $q_{t}$ and increasing in $z_{t}$, where $q_{t}$ is output and $z_{t} \in[\underline{z}, \bar{z}]$ are persistent demand shocks. The $z_{t}$ process is a time invariant monotone Markov process which satisfies the Monotone Mixing Condition of Theorem 2. Points $\underline{z}$ and $\bar{z}$ are in the support of the invariant distribution of the $z_{t}$ process. The interest rate is constant at $r$.

There are constant returns to scale with output, $q_{t}$ constrained by capacity, i.e. if $k_{t}$ is the capacity at time $t$ and $q_{t}$ the output, then $q_{t} \leq k_{t}$. The cost of increasing capacity in one period from $k_{t}$ to $k_{t+1}$ is given by $k_{t} h\left(k_{t+1} / k_{t}\right)$, where $h$ is assumed convex, nonnegative and increasing, with $h(1)>0$. Assume that $D(\bar{q}, \bar{z}) \leq 0$ for some finite $\bar{q}\left({ }^{10}\right)$, so that there exists some $\bar{k}$ such that if $k_{t} \leq \bar{k}$, then $k_{t+1} \leq \bar{k}$, and assume that $h^{\prime}(\infty)<D(0, \underline{z})$ so that $k_{t}$ does not remain at zero forever.

As there are constant returns, only the industry aggregate capacity matters and not the distribution of capacity over firms. In consequence, only the case of a single price taking firm needs to be considered. The firm maximizes its expected present value.

Let

$$
\mathrm{s}(\mathrm{k}, \mathrm{z})=\int_{0}^{\mathrm{k}} \mathrm{D}(\mathrm{q}, \mathrm{z}) \mathrm{dq}
$$

be the area under the inverse demand curve. Let $m=s(\bar{k}, \bar{z})$. Then the surplus function $\mathrm{s}:[0, \mathrm{~K}] \times[\underline{z}, \mathrm{z}] \rightarrow[0, \mathrm{~m}]$ is strictly concave in $\mathrm{k}$, strictly increasing and continuous in both its arguments, positive and bounded.

Lucas and Prescott(1971) show that the competitive equilibrium allocation solves the program 
$\max E\left[\sum_{t=0}^{\infty} \frac{1}{(1+\mathrm{r})^{\mathrm{t}}}\left[\mathrm{s}\left(\mathrm{k}_{\mathrm{t}}, \mathrm{z}_{\mathrm{t}}\right)-\mathrm{k}_{\mathrm{t}} \mathrm{h}\left(\mathrm{k}_{\mathrm{t}+1} / \mathrm{k}_{\mathrm{t}}\right)\right]\right]$

subject to $0 \leq q_{t} \leq k_{t}$ and $k_{0}$ given.

The state space for this problem is $S=[0, \mathrm{k}] \times[\mathrm{z}, \mathrm{Z}]$. It is straightforward to verify by using now standard dynamic programming techniques that the optimal policy is generated by a stationary plan $k_{t+1}=g\left(k_{t}, z_{t}\right)$, where $g$ is strictly increasing and continuous. Further, the (gross) growth rate of capacity, namely $\mathrm{g}(\mathrm{k}, \mathrm{z}) / \mathrm{k}$, is strictly decreasing in $\mathrm{k}$ and increasing in $\mathrm{z}$.

Existence of an invariant distribution is an immediate application of Corollary 5 since. the Markov process generated by $\mathrm{g}$ is monotone and $\mathrm{S}$ is a compact metric space with minimal element $(0, \underline{z})$. A stronger result can be obtained, namely that Theorem 2 applies to this case. We will now check that the Monotone Mixing Condition is satisfied.

Let $k(z)$ be the function that for each $z$ gives the $k$ which satisfies

$$
\mathrm{k}(\mathrm{z})=\mathrm{g}[\mathrm{k}(\mathrm{z}), \mathrm{z}]
$$

or, equivalently, the growth rate equals one, i.e.

$$
\mathrm{g}[\mathrm{k}(\mathrm{z}), \mathrm{z}] / \mathrm{z}=1
$$

The growth rate is a continuous function of $k$ and $z$, strictly decreasing in $k$ and increasing in $z$, and goes to infinity as $\mathrm{k}$ goes to zero and to zero as $\mathrm{k}$ goes to $\mathrm{k}$. In consequence the function $\mathrm{k}($.$) can be shown to be well defined, continuous and strictly increasing.$

We now show that the point $s^{*}=\left(k^{*}, z^{*}\right)$, where $k^{*}=(\underline{k}+\bar{k}) / 2$ and $z^{*}=(\underline{z}+\bar{z}) / 2$ must satisfy the Monotone Mixing Condition of Theorem 2. For $k=\underline{k}$ and $z=\underline{z}$ the growth rate is one. 
For any larger $\mathrm{k}$ and $\mathrm{z}=\underline{\mathrm{z}}$, the probability of reaching any neighborhood of $\underline{\mathrm{k}}$ is positive. Thus even if $s_{0}=\bar{s}=(\bar{k}, \bar{z})$ the probability of reaching the set $\left\{s \in S: s \leq s^{*}\right\}$ is positive. By a similar argument, if $s_{0}=\underline{s}=(\underline{k}, \underline{z})$ the probability of reaching the set $\left\{s \in S: s \geq s^{*}\right\}$ is also positive. Hence by Theorem 2 there is a unique invariant distribution for the state $s=(k, z)$ and $P^{n}(s,$.$) converges$ to it for all s.

Another economic problem to which the theorems can be applied is the stochastic growth model (Brock and Mirman, 1972). The problem is:

$$
\max E\left\{\sum_{t=0}^{\infty} \beta^{\mathrm{t}} \mathrm{u}\left(\mathrm{c}_{\mathrm{t}}\right)\right\} \text { where } 0<\beta<1,
$$

subject to

$$
k_{t+1}+c_{t} \leq z_{t} f\left(k_{t}\right)
$$

and $c_{t}, k_{t+1} \geq 0$ for all $t$.

The utility function $u: R_{+} \rightarrow R_{+}$is strictly increasing, strictly concave and differentiable with $\mathrm{u}^{\prime}(0)=\infty$. The production function $\mathrm{f}: \mathrm{R}_{+} \rightarrow \mathrm{R}_{+}$is strictly increasing, concave and differentiable. The $z_{\mathrm{t}}$ are i.i.d. random variables with support $[1, \mathrm{z}]$ and probability distribution $\psi$. For some $\bar{k}>0$ and maximal shock $\bar{z}$, it is also assumed that $\bar{k}=\bar{z} f(\bar{k})$. Finally $\beta f^{\prime}(0)$ is assumed to be greater than 1 and $f(0)>0 .(11)$

The optimality equation corresponding to this recursive problem is:

$$
\mathrm{v}(\mathrm{k}, \mathrm{z})=\sup \left\{\mathrm{u}[\mathrm{zf}(\mathrm{k})-\mathrm{y}]+\beta \int \mathrm{v}\left(\mathrm{y}, \mathrm{z}^{\prime}\right) \psi\left(\mathrm{d} \mathrm{z}^{\prime}\right)\right.
$$

subject to

$$
0 \leq y \leq z f(k) .
$$


Some standard results for this problem are:

(i) A unique, concave, continuous function $\mathrm{v}:[0, \mathrm{~K}] \times[1, \mathrm{z}] \rightarrow \mathrm{R}$ exists that solves the functional equation (7) and it is the optimal return function for the problem above.

(ii) There is a unique policy function $\mathrm{g}:[0, \mathrm{~K}] \times[1, \mathrm{z}]$ such that $\mathrm{k}_{\mathrm{t}+1}=\mathrm{g}\left(\mathrm{k}_{\mathrm{t}}, \mathrm{z}_{\mathrm{t}}\right)$ attains the supremum in (7) and it generates the optimal plan. The function is increasing in both its arguments and continuous.

(iii) The consumption function $c(k, z)=z f(k)-g(k z)$ is increasing in both its arguments.

(iv) The value function is differentiable in $\mathrm{k}$ with

$$
\frac{\partial \mathrm{v}}{\partial \mathrm{k}}(\mathrm{k}, \mathrm{z})=\mathrm{u}^{\prime}[\mathrm{c}(\mathrm{k}, \mathrm{z})] \mathrm{zf}^{\prime}(\mathrm{k}) \text {. }
$$

For this problem the state space is $S=[0, \mathrm{k}] \times[1, \bar{z}]$, a compact set. Given the monotonicity of $g$ and the independence of $z_{t}$, the Markov process on the state is monotone. As $\mathrm{S}$ has a minimal element, by Theorem 1 the process has an invariant distribution.

$$
\text { Let } \begin{aligned}
\mathrm{s}^{*} & =\left(\mathrm{k}^{*}, \mathrm{z}^{*}\right), \text { where } \\
\mathbf{z}^{*} & =\int \mathrm{z} \psi(\mathrm{dz})
\end{aligned}
$$

and let $k^{*}$ be the solution to $\frac{1}{\beta}=z^{* f^{\prime}}\left(k^{*}\right)$.

We now show that s* satisfies the Monotone Mixing Condition of Theorem 2.

Let $\left\{k_{n}\right\}$ be the sequence obtained from the optimal policy rule $g\left(k_{n}, 1\right)$ starting at $\mathrm{k}_{\mathrm{o}}=\overline{\mathrm{k}}$; i.e the sequence of capital stocks starting at the maximal sustainable capital stock and provided thereafter the productivity shock is at its minimum. By monotonicity of the optimal policy rule $g$, the sequence $\left\{k_{n}\right\}$ is decreasing and since it is bounded $k_{n} \rightarrow b \in[\underline{k}, k]$. Further, continuity of $\mathrm{g}$ implies $\mathrm{b}=\mathrm{g}(\mathrm{b}, 1)$. For any $\delta>0, \psi([1,1+\delta))>0$, since $\{1\}$ is in the support of $\psi$. This, together with the continuity of $g$ imply that the probability of eventually being in any 
neighborhood of $b$ is positive.

The first order condition in the optimization problem if $s=(b, 1)$ is

$$
\mathrm{u}^{\prime}\left[\mathrm{c}(\mathrm{b}, 1]=\beta \int \frac{\partial \mathrm{v}}{\partial \mathrm{k}}\left(\mathrm{b}, \mathrm{z}^{\prime}\right) \psi\left(\mathrm{d} \mathrm{z}^{\prime}\right)=\beta \mathrm{f}^{\prime}(\mathrm{b}) \int \mathrm{u}^{\prime}\left[\mathrm{c}\left(\mathrm{b}, \mathrm{z}^{\prime}\right)\right] \mathrm{z}^{\prime} \psi\left(\mathrm{d} \mathrm{z}^{\prime}\right)\right.
$$

as under the optimal plan $b=g(b, 1)$. But $u^{\prime}\left[c\left(b, z^{\prime}\right)\right] \leq u^{\prime}[c(b, 1)]$ with strict inequality if $z^{\prime}>1$ given that $u$ is strictly concave and $c$ is strictly increasing in $z$. Thus

$$
\mathrm{u}^{\prime}[\mathrm{c}(\mathrm{b}, 1)]<\beta \mathrm{f}^{\prime}(\mathrm{b}) \mathrm{u}^{\prime}[\mathrm{c}(\mathrm{b}, 1)] \int \mathrm{z}^{\prime} \psi\left(\mathrm{dz} \mathrm{z}^{\prime}\right)
$$

or

$$
\frac{1}{\beta}<z^{*} f^{\prime}(b)
$$

But since $\mathrm{f}$ was assumed to be strictly concave and $\frac{1}{\beta}=\mathrm{z}^{*} \mathrm{f}^{\prime}\left(\mathrm{k}^{*}\right), \mathrm{b}<\mathrm{k}^{*}$.

By an analogous argument, the limit of the sequence generated from $\mathrm{k}_{\mathrm{o}}=0$ and $k_{n+1}=g\left(k_{n}, \bar{z}\right)$, denoted by a, must exceed $k^{*}$. Thus starting at $\underline{s}=(0,1)$, the probability that $k_{t}$ eventually is in any neighborhood of a is positive and therefore the probability of reaching the set $\left[\mathrm{k}^{*}, \mathrm{k}\right]$ is positive as well. As $\psi\left(\left[\mathrm{z}^{*}, \mathrm{Z}\right]\right)$ and $\psi\left(\left[1, \mathrm{z}^{*}\right]\right)$ are both positive, the Monotone Mixing Condition is satisfied. 


\section{NOTES}

1 By the Feller type conditions' approach we mean the approach which requires for any continous and bounded function $f$,

$$
\operatorname{Tf}(\mathrm{s})=\int f\left(\mathrm{~s}^{\prime}\right) \mathrm{P}\left(\mathrm{s}, \mathrm{ds} \mathrm{s}^{\prime}\right)
$$

to be a continuous and bounded function. The mapping of the space of measures into itself induced by $\mathrm{P}$ is identified to the adjoint operator of $\mathrm{T}$, and standard fixed point arguments are thereof applied. Rosenblatt(1971) has a section devoted to this approach. Other references can be found in Futia(1982). Criteria for uniqueness of the invariant measure within this framework are discussed in Sine(1968) and Jamison(1964 and 1965).

2 A fixed point argument based on stochastic dominance ordering is presented in Green, McKelvey and Packel, 1981. They develop a technique for proving existence and obtaining bounds for the concentration of a stationary distribution for a given Markov process on the basis of comparisons, via stochastic dominance, with a different Markov process, having a known stationary distribution. Their argument also requires a continuity condition on the Markov process and the existence of an invariant distribution for the referential process.

3 All the results in this section hold if $\mathrm{S}$ is a complete, metric separable space (Polish space). In particular, Propositions 1 and 2 and lemma 4 hold for $S$ Polish, since if $\mu$ is a finite measure on $(\mathrm{S}, \mathcal{S})$, where $\mathcal{S}$ is the Borel $\sigma$-algebra of subsets of $\mathrm{S}$, then $\mu$ is tight , i.e for any $\epsilon>0$ there is a compact subset of $S, K_{\epsilon}$, such that $\mu\left(S \backslash K_{\epsilon}\right)<\epsilon$, and thus the approximation procedures used in these propositions extend to Polish spaces. Since the theorems in the paper we require compactness of the set of measures and given the strong connection there is between compactness of sets of measures and compactness of the underlying measure space, we have decided to restrict our attention to compact metric $S$.

4 This ordering does not give $\mathcal{H}(S)$ a lattice structure. To see this consider the following example, provided to us by N. Stokey:

Let $S=[0,1] \times[0,1]$ and define the measures $\mu$ and $\nu$ by

and

$$
\mu\{(0,0)\}=\mu\{(1,1)\}=\frac{1}{2}
$$

$$
\nu\{(0,1)\}=\nu\{(1,0)\}=\frac{1}{2} .
$$

For $\lambda$ to be an upper bound for $\{\mu, \nu\}$ it is necessary that:

i) $\lambda(\{(1,1)\}) \geq \frac{1}{2}$

and ii) $\lambda(F)=1$ where $F=\{(x, y): x+y \geq 1\}$.

$$
\begin{aligned}
& \text { Let } \lambda_{1}(\{(1,0)\})=\lambda_{1}(\{(1,1)\})=\frac{1}{2} \text { and } \\
& \lambda_{2}(\{(0,1)\})=\lambda_{2}(\{(1,1)\})=\frac{1}{2} .
\end{aligned}
$$


Clearly, $\lambda_{1}$ and $\lambda_{2}$ are upper bounds for $\{\mu, \nu\}$.

Suppose $\lambda$ is an upper bound for $\{\mu, \nu\}$ and $\lambda \leq \lambda_{1}$. Then it must be the case that $\lambda$ concentrates its mass in $\{1\} \times[0,1]$ since otherwise $\lambda \leq \lambda_{1}$ would be violated by the monotone function $\mathrm{f}(\mathrm{x}, \mathrm{y})=\mathrm{x}$ But if $\lambda\left(\{(1,0)\} \neq \frac{1}{2}\right.$ a similar argument would show that $\lambda$ cannot be a lower bound for $\lambda_{1}$. Hence $\lambda=\mu$.

This same argument shows that the only upper bound for $\{\mu, \nu\}$ that is a lower bound for $\lambda_{2}$ is $\lambda_{2}$. But $\lambda_{1}$ and $\lambda_{2}$ are not comparable under $\succeq$, so there is no lower bound for the set of upper bounds of $\{\mu, \nu\}$.

5 Kamae, Krengel and O'Brien (1977) have an interesting paper in which they show , using a theorem from Strassen (1965) that the stochastic ordering is a closed ordering and that an increasing sequence in this ordering converges. This is basically what we need and we could have taken their result as given and proved directly Proposition 4 . We have followed an alternative more constructive approach and thought convenient to make it explicit. We believe that it may aid the intuition in the study of monotone Markov processes and that some intermediate approximation results obtained may be useful by themselves. To the interested reader we highly recommend the above mentioned papers.

6 Let $\left\{\mathrm{f}_{\mathrm{n}}\right\}$ be a countable set in $\mathrm{C}(\mathrm{S})$ including the unit function. A metric corresponding to the weak* topology of $\mathcal{H}(\mathrm{S})$ is defined by

$$
\rho(\mu, \nu)=\sum_{\mathrm{j}=1}^{\infty} \frac{\mid\left\langle\mathrm{f}_{\mathrm{j}} \mathrm{d} \mu-\left\langle\mathrm{f}_{\mathrm{j}} \mathrm{d} \mu\right|\right.}{1+\left|\mathrm{f}_{\mathrm{j}} \mathrm{d} \mu-\int \mathrm{f}_{\mathrm{j}} \mathrm{d} \mu\right|} .
$$

7 The construct when $S c R^{n}$ is a rectangle, i.e $S=\left\{s \in R^{n}: a \leq s \leq b\right\}$ for some elements a and $\mathrm{b}$ in $\mathrm{R}^{\mathrm{n}}$ is illustrative of the way this

function is obtained. For any $\mathrm{s} \epsilon \mathrm{S}$ let $\mathrm{g}(\mathrm{s})=\rho\left(\mathrm{s}, \mathrm{C}_{\leq}\right)$, where $\rho$ is the Hausdorff distance. let $\mathrm{f}(\mathrm{s})=\min \left\{1, \frac{\mathrm{g}(\mathrm{s})}{\rho\left(\mathrm{D}_{\geq}, \mathrm{C}_{\leq}\right)}\right\}$. Note that $\mathrm{f}$ is continuous since $\mathrm{g}$ is continuous in $\mathrm{s}$. For $\mathrm{s} \epsilon \mathrm{C}_{\leq}$, $g(s)=0$, so $f(s)=\overline{0}$. For $s \in D_{\geq} g(s) \geq \rho\left(D_{\geq}, C_{\leq}\right)$and hence $f(s)=1$. If $s \geq s^{\prime}$, then $f(s) \geq f\left(s^{\prime}\right)$ trivially if $s \epsilon \mathrm{D}_{\geq}$or $s^{\prime} \epsilon \mathrm{C}_{\leq}$. If this is not the case, we will show that for any $z \epsilon \mathrm{C}_{\leq}$, we can find $z^{\prime}$ in $\mathrm{C}_{\leq}$such that $\rho\left(\mathrm{s}^{\prime}, \mathrm{z}^{\prime}\right) \leq \rho(\mathrm{s}, \mathrm{z})$. So let $z \in \mathrm{C}_{\leq}$Let $\mathrm{J}=\left\{\mathrm{j} \epsilon(1,2, \ldots, N\}: \mathrm{s}_{\mathrm{j}}^{\prime} \leq \mathrm{z}_{\mathrm{j}}\right\}$. Define $\mathrm{z}^{\prime}$ by $z_{j}^{\prime}=\min \left\{z_{j}, s_{j}^{\prime}\right\}$. Since $a \leq z^{\prime} \leq z, z^{\prime} \epsilon C_{\leq}$. Then

$\rho\left(s^{\prime}, z^{\prime}\right)=\left(\sum_{j \notin J}\left(s_{j}^{\prime}-z_{j}\right)^{2}\right)^{1 / 2} \leq\left(\sum_{j \notin J}\left(s_{j}-z_{j}\right)^{2}\right)^{1 / 2} \leq \rho(s, z)$.

This shows that $\rho\left(z^{\prime}, \mathrm{C}_{\leq}\right) \leq \rho\left(z, \mathrm{C}_{\leq}\right)$, and thus $\mathrm{f}(\mathrm{s}) \geq \mathrm{f}\left(\mathrm{s}^{\prime}\right)$.

8 If $\mathrm{S}$ is compact, by the Riesz representations theorem (see Dunford and Schwartz,1958 IV.6.3.) the dual space of the set $C(S)$ of continuous and bounded functions on $S$ can be identified with the space of measures on $(S, \mathcal{S})$. The space of all measures uniformly bounded by $\mathrm{m}$ is closed in the weak* topology and bounded in the strong topology. By the Banach-Alaoglu theorem (see Corollary V.4.3. in Dunford and Shwartz) this set is compact.

$9 \quad$ Similar results were obtained by Dubins and Freedman [1966] but restricting the state space to the unit interval and with continuity requirements on the process. In their paper they define a metric on the space of measures which gives a topology stronger than the 
weak* topology, and use a contraction mapping argument. This result was extended to a rectangle in $\mathrm{R}^{\mathrm{k}}$ by Bhattacharya and Lee, 1986 .

10 If there is a variable input that enters in fixed proportion, with pioportionality factor $\lambda$, into production, then this assumption could be replaced by $D(\bar{q}, \bar{z})-\lambda \bar{q} \leq 0$.

11 The assumption $f(0)>0$ simplifies the uniqueness argument. If $f(0)=0$ then the measure which concentrates all the mass in the point set $\{0\}$ will also be an invariant distribution and, under general conditions, there will exist only one invariant distribution besides this trivial one. Monotonicity arguments can still be applied. 


\section{REFERENCES}

Ash, R.: Real Analysis and Probability. New York: Academic Press, 1972.

Bhattacharya, R.N. and Oesook Lee: "Existence of Unique Invariant Probabilities and Functional Central Limit Theorems for Some Special Classes of Markov Processes", Proceedings of the First World Congress of the Bernoulli Society, Tashkent, Sept 1986.

Billingsley,P.: Convergence of Probability Measures. New York: J. Wiley \& Sons, 1968

Brock W., and L. Mirman: "Optimal Growth and Uncertainty: The discounted case",Journal of Economic Theory, 4(1972),479-513

Donaldson J.B., and R. Mehra: "Stochastic Growth with Correlated Production Shocks", Journal of Economic Theory 29(1983), 282-312.

Dubins, L.and D.Freedman:"Invariant Probabilities for Certain Markov Processes", Annals of Mathematical Statistics, 1966 pp.837-848....

Dugundji, J. and A. Granas: Fixed point theory. Vol. 1. Warsaw: PWN-Polish Scientific Publishers, 1982.

Dunford, N. and Schwartz, J. Linear Operators. Part I. New York: Interscience, 1958.

Futia, C.:."Invariant distributions and the limiting behavior of Markovian economic models. Econometrica 50(1982), 377-408.

Green, E., R. McKelvey and E. Packel: "Methods for Comparison of Markov Processes by Stochastic Dominance", Social Science Working Paper 395, California Institute of Technology, July 1981.

Hopenhayn(1986) "A Competitive, Stochastic model of Entry and Exit to an Industry", Department of Economics, unpublished manuscript, 1986.

Jamison, B.: "Asymptotic Behavior of Successive Iterates of Continuous Functions Under a Markov Operator", Journal of Mathematical Analysis and Applications, 9(1964),203-214.

Trangatic : "Ergodic Decomposition Induced by Certain Markov Operators", Transactions of the American Mathematical Society, 117(1965), 451-468.

Jovanovic B.: "Job Matching and the Theory of Turnover", Journal of Political Economy 87(1979), 972-990.

Kamae, T., U. Krengel, and G.L. O'Brien: "Stochastic Inequalities on Partially Ordered Spaces", The Annals of Probability 5(1.977), 899-912.

Kelley, J: General Topology. New York: Van Nostrand, 1955.

Lucas R.E.: "Asset Prices in an Exchange Economy", Econometrica, 46(1978): 1429-1444. 
Lucas R.E., and E.C. Prescott: "Investment Under Uncertainty", Econometrica, 39(1971), $659-681$.

Journal of Economic Theory, 7(1974): 188-209.

Majumdar M., T. Mitra, and Y. Nyarko: "Dynamic Optimization Under Uncertainty: Non-convex feasible set", Department of Economics, Cornell University, 1985.

Manuelli R.: "A General Equilibrium Model of International Credit Markets", unpublished manuscript, 1985.

Marimon R.: "General Equilibrium and Growth under Uncertainty: The Turnpike property", Discussion paper \#624, Center for Mathematical Studies in Economics and Management, August 1984.

Nachbin L.: Topology and Order. New York: Van Nostrand, 1965.

Parthasarathy, K.R.: Probability Measures on Metric Spaces. New York: Academic Press, 1967.

Rosenblatt M.: Markov Processes.Structure and Asymptotic Behavior. New York: Springer Verlag, 1971.

Sine, R.: "Geometric Theory of a Single Markov Operator", Pacific Journal of Mathematics, $27(1968), 155-166$.

Strassen, V.: "The Existence of Probability Measures with Given Marginals", Annals of Mathematical Statistics 36(1965), 423-439. 